\title{
Welche Nichtraucher COPD gefährdet sind
}

\author{
Auch Nichtraucher erkranken an COPD. Welche Menschen besonders gefährdet sind, haben ka- \\ nadische Forscher nun untersucht.
}

Nahezu jeder dritte COPD-Patient ist Nichtraucher, die meisten davon sind Frauen. Bislang gibt es nur wenige Untersuchungen zu den Risikofaktoren der Nikotinabstinenten. Unklar ist zudem, ob die Atemnot von Rauchern und Nichtrauchern mit COPD unterschiedlich behandelt werden sollte.

Wan Tan von der University of British Columbia, Vancouver, und Kollegen haben Daten von 5.176 Personen ab 40 Jahren der populationsbasierten, prospektiven „Canadian Cohort of Obstructive Lung Disease Study“ (CanCOLD-Studie) analysiert (Thorax 2015;70:822-29). Verglichen wurden geschlechtsspezifische Charakteristika sowie der Schweregrad der Atemwegsobstruktion von Nichtrauchern (Never-Smoker: Lebenszeitexposition $<1 / 20$ Packungsjahr) und Rauchern (Ever-Smoker).

$6,4 \%$ der Nichtraucher litten unter einer COPD $\left(\mathrm{FEV}_{1} / \mathrm{FVC}<\right.$ 5. Perzentile), 43\% dieser Gruppe unter mittelgradiger bis schwerer Atemnot. Unter den Rauchern lag die COPD-Quote bei 15,3\% (62\% mit mittelgradiger bis schwerer Atemnot). Insgesamt machten die Nichtraucher 29\% der COPD-Patienten aus.

Als unabhängige, geschlechtsneutrale COPD-Risikofaktoren ermittelten Tan und Kollegen in der multivariaten Analyse sowohl für Nichtraucher als auch für Raucher ein höheres Lebensalter, eine Asthmaerkrankung sowie einen geringen Bildungsgrad. Risikofördernd bei den Nichtrauchern waren zudem Klinikaufenthalte in der Kindheit wegen Atemwegserkrankungen.

\section{Unterschiede bei den Geschlechtern}

Speziell bei nicht rauchenden Frauen erwiesen sich Passivrauchen sowie eine jahrelange Exposition gegenüber biogenen Heizmaterialien (wie Kohle oder Holz über mindestens zehn Jahre) als COPD fördernd. Innerhalb der Rauchergruppe zeigten sich dagegen keine geschlechtsspezifischen Unterschiede hinsichtlich möglicher Risikofaktoren. Dafür addierten sich hier die Folgen des Zigarettenrauchens entsprechend der $\mathrm{Pa}-$ ckungsjahre. In einer Substudie wurde zudem deutlich: Unabhängig vom Raucherstatus zeigten sich gegenüber Studienteilnehmern ohne COPD vermehrt charakteristische respiratorische Symptome wie Exazerbationen, chronischer Husten, Auswurf, Atemnot und Wheezing sowie ein erhöhter Residual-/ Gesamtlungenvolumen-Quotient.

Der pulmonale Transferkoeffizient für Kohlenmonoxid (DLCO/Va) dagegen war nur bei Rauchern mit COPD reduziert. Ebenfalls nur in der Rauchergruppe gehäuft traten eine erhöhte Gesamtlungenkapazität sowie Emphyseme im CT auf.

Ein zentrales Ergebnis der Studie, so die Autoren, sei der unabhängige Risikofaktor „Asthma", der für Raucher (30\%) und Nichtraucher (36\%) gleichermaßen erkennbar war. Diese Kombination könne als „Asthma-COPD-Overlap-Syndrom" bezeichnet werden.

Auch die Geschlechtsunterschiede hinsichtlich der Risikofaktoren seien interessant. Zwar sei ein Zusammenhang zwischen Biokraftstoffen und COPD bei Frauen in Entwicklungsländern bekannt, nicht aber aus Nordamerika oder Westeuropa. Hier müssten die Lebensumstände in weiteren Studien erneut überprüft werden.

Die Studie mache deutlich, so Tan und Kollegen, wie häufig auch Nichtraucher an einer COPD erkranken, und weise auf deren allgemeine und spezielle Risikofaktoren hin. Zudem erlaube sie einen ersten Blick auf mögliche phänotypische Unterschiede der COPD zwischen Rauchern und Nichtrauchern. Mit ihrer Kenntnis könnten gegebenenfalls gezieltere Therapien möglich werden.

Christine Starostzik

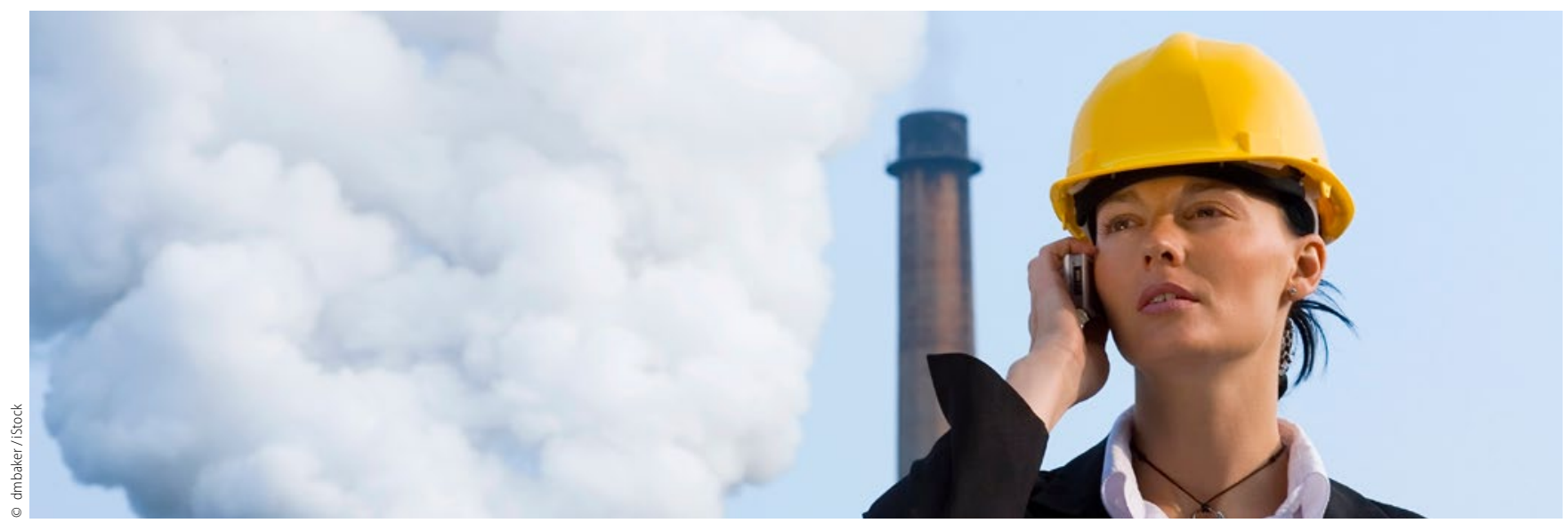

Eine jahrelange Exposition gegenüber biogenen Heizmaterialien wie Kohle erwies sich gerade bei nicht rauchenden Frauen als ein Risikofaktor für eine COPD. 